\title{
Frontier detectors for frontier physics, PM2015 Performance of pile-up mitigation techniques for jets in pp collisions with the ATLAS detector
}

The large rate of multiple simultaneous proton-proton interactions, or pile-up, generated by the Large Hadron Collider in Run I required the development of many new techniques to mitigate the adverse effects of these conditions. The methods employed to correct for the impact of pile-up on jet energy, shapes and multiplicity are here presented. They include energy correction techniques based on estimates of the average pile-up energy density and jet-to-vertex association techniques. Extensions of these methods to reduce the impact of pile-up on jet shapes use both subtraction and grooming procedures. Prospects for pile-up suppression at the HL-LHC are also shown.

Pile-up jet tagging using tracking

\section{information}

This pile-up jet tagging technique relies on the possibility to

- associate tracks to jets

- tag jets based on the track origin

It uses a combination of discriminants,

such as the jet vertex fraction (JVF) and the charged energy fraction of the jet $\left(R_{\mathrm{pT}}\right)$

$J V F=\frac{\sum_{k} p_{T}^{t r k_{k}}\left(P V_{0}\right)}{\sum_{k} p_{T}^{t r k_{k}}} \quad R_{p T}=\frac{\sum_{k} p_{T}^{t r k k_{k}}\left(P V_{0}\right)}{p_{T}^{j e t}}$
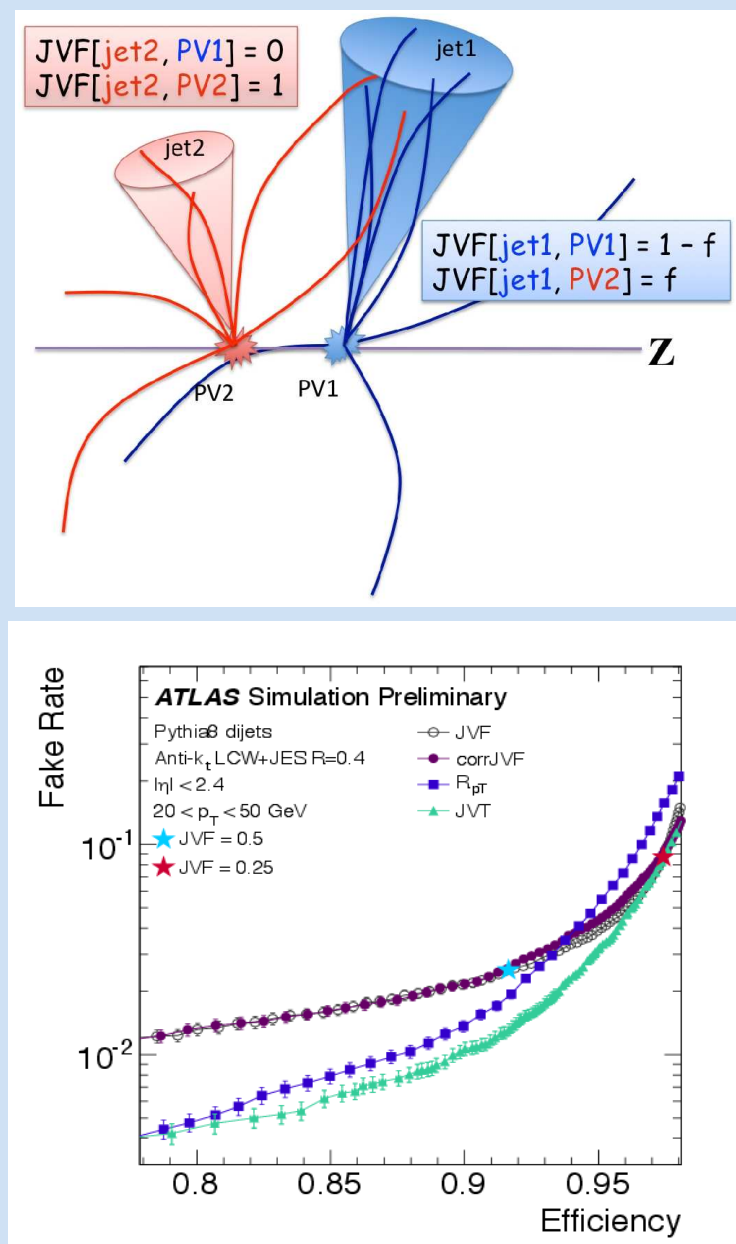

The performance of the resulting Jet Vertex Tagger (JVT) shows 1\% fake rate from pile-up jets for a hardscatter jet efficiency of 0.9 .

ATLAS-CONF-2014-018

\section{Pile-up subtraction to correct jet energy and shapes}

ATLAS-CONF-2013-083

The Jet Area correction estimates the average pileup $\mathrm{p}_{\mathrm{T}}$ density in the event and corrects the jet $p_{\mathrm{T}}$ according to its area. It significantly reduces the dependence on pile-up of the energy resolution.

$$
p_{T}^{\text {corr }}=p_{T}-\rho \times A_{T}-\alpha\left(N_{P V}-1\right)-\beta\langle\mu\rangle \rightarrow \begin{aligned}
& \text { Correction of the residual pile-up } \\
& \text { dependence due to local effects. }
\end{aligned}
$$
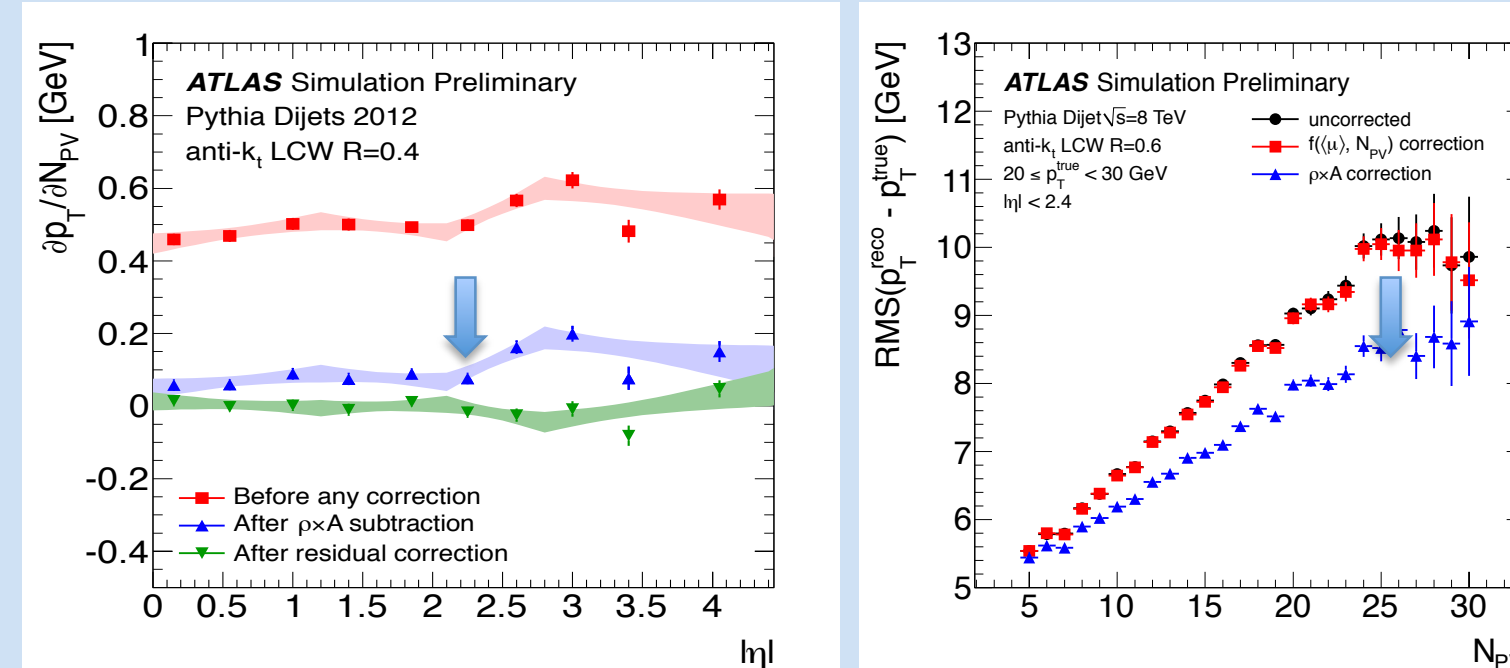

ATLAS-CONF-2013-085

The pile-up shape subtraction method evaluates the sensitivity of the jet shape to variations in infinitesimal soft energy depositions. Better stability with respect to to pile-up conditions is obtained for the ${ } \mathrm{d}_{12}$ splitting scale, used to distinguish between boosted top and light jets.

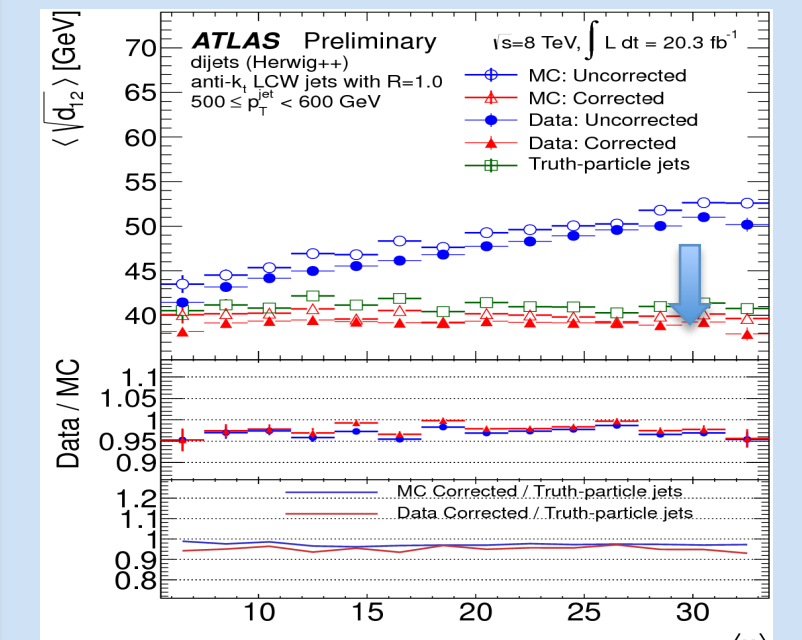

\section{Pile-up suppression with jet substructure}

Jet substructure techniques are designed to improve the mass resolution of hadronically decaying boosted particles. Jet Trimming techniques reduce effective jet area rejecting low-momentum sub-jets arising from pile-up. They reduce the mass and energy dependence from pile-up. These techniques can be combined with tracking information by tagging pile-up sub-jets using tracks. JHEP09(2013)076
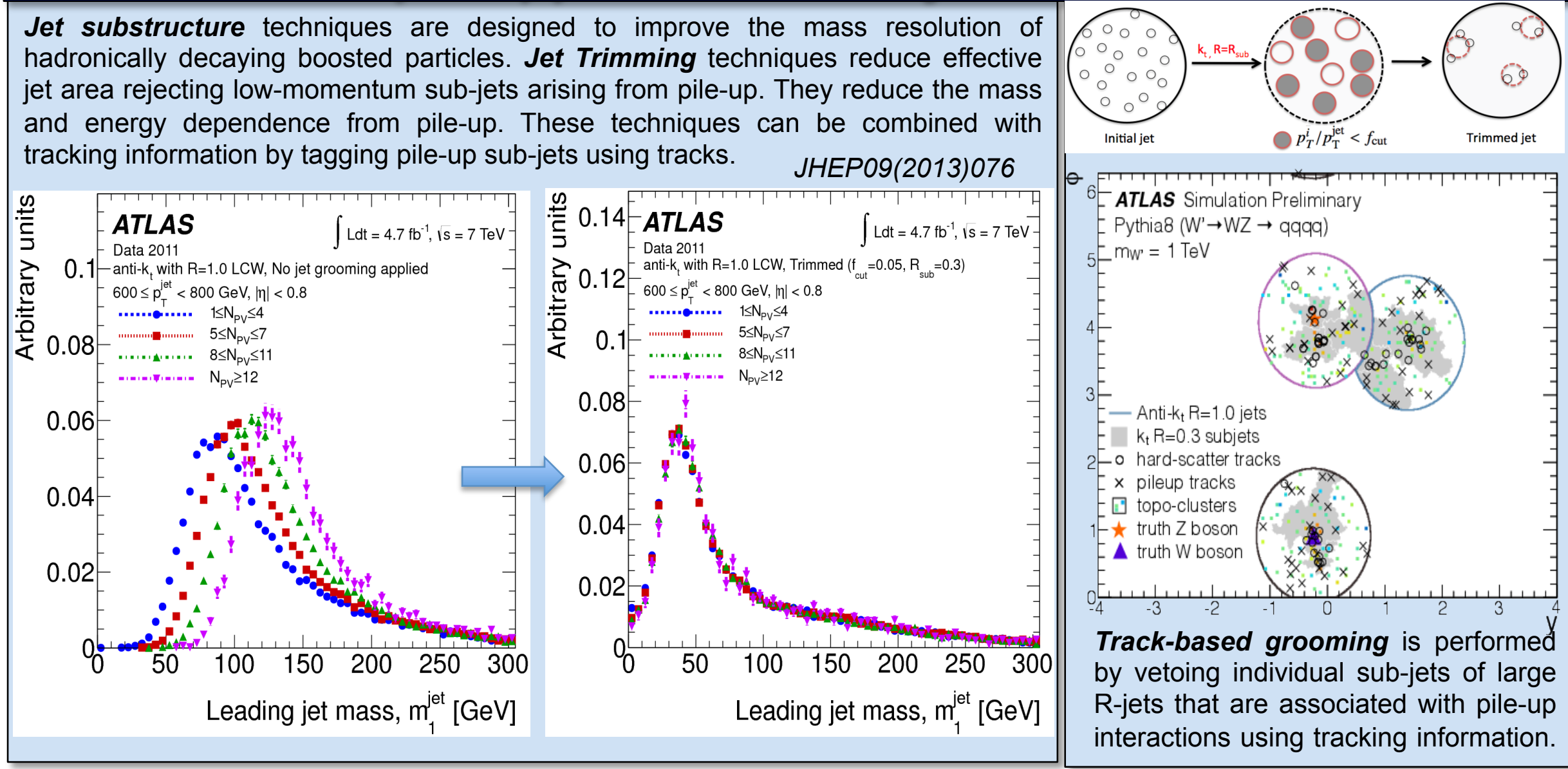

Track-based grooming is performed by vetoing individual sub-jets of large $\mathrm{R}$-jets that are associated with pile-up interactions using tracking information.

\section{Pile-up suppression at HL-LHC}

The design luminosity of $5 \times 10^{34} \mathrm{~cm}^{-2} \mathrm{~s}^{-1}$ planned for the High Luminosity $(\mathrm{HL})$ LHC will imply an average pile-up of 140 interactions per bunch crossing. Techniques using tracking information and substructure information combined with the Jet Area subtraction can successfully cope with such harsh conditions. Simulation studies show that the dependence on pile-up of jet multiplicity and jet mass can be significantly reduced
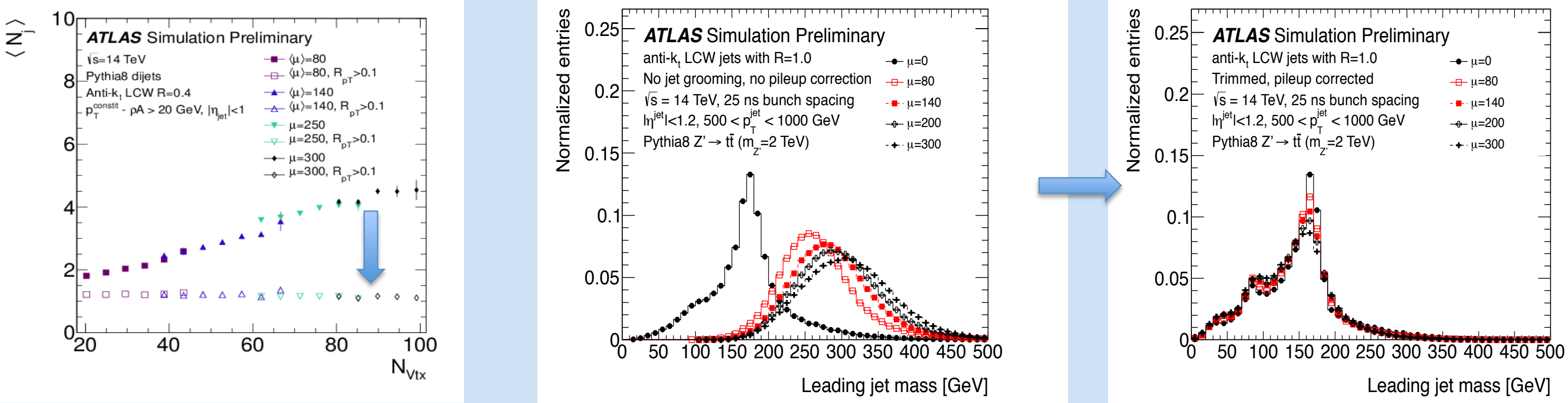